\title{
Loss of DDHD2, whose mutation causes spastic paraplegia, promotes reactive oxygen species generation and apoptosis
}

Tomohiro Maruyama', Takashi Baba 1,7, Yuki Maemoto', Chikako Hara-Miyauchi², Minami Hasegawa-Ogawa², Hirotaka James Okano², Yuki Enda', Kei Matsumoto', Nagisa Arimitsu, , Kazuki Nakao ${ }^{3}$, Hiroshi Hamamoto ${ }^{4,9}$, Kazuhisa Sekimizu,9, Takayo Ohto-Nakanishi ${ }^{5}$, Hiroki Nakanishi', Takeshi Tokuyama', Shigeru Yanagi',

Mitsuo Tagaya (10 ${ }^{1}$ and Katsuko Tani ${ }^{1}$

\begin{abstract}
DDHD2/KIAA0725p is a mammalian intracellular phospholipase $A_{1}$ that exhibits phospholipase and lipase activities. Mutation of the DDHD2 gene causes hereditary spastic paraplegia (SPG54), an inherited neurological disorder characterized by lower limb spasticity and weakness. Although previous studies demonstrated lipid droplet accumulation in the brains of SPG54 patients and DDHD2 knockout mice, the cause of SPG54 remains elusive. Here, we show that ablation of DDHD2 in mice induces age-dependent apoptosis of motor neurons in the spinal cord. In vitro, motor neurons and embryonic fibroblasts from DDHD2 knockout mice fail to survive and are susceptible to apoptotic stimuli. Chemical and probe-based analysis revealed a substantial decrease in cardiolipin content and an increase in reactive oxygen species generation in DDHD2 knockout cells. Reactive oxygen species production in DDHD2 knockout cells was reversed by the expression of wild-type DDHD2, but not by an active-site DDHD2 mutant, DDHD2 mutants related to hereditary spastic paraplegia, or DDHD1, another member of the intracellular phospholipase $A_{1}$ family whose mutation also causes spastic paraplegia (SPG28). Our results demonstrate the protective role of DDHD2 for mitochondrial integrity and provide a clue to the pathogenic mechanism of SPG54.
\end{abstract}

\section{Introduction}

Hereditary spastic paraplegia (HSP) is a diverse group of neurological disorders characterized by lower limb spasticity and weakness ${ }^{1,2}$. These symptoms are due to lengthdependent axonopathy of corticospinal motor neurons, sometimes associated with a loss of cortical neurons and anterior horn cells ${ }^{3}$. The severity of HSP is variable, and the age at onset ranges from early childhood to late in life. To date, nearly 80 genes or loci have been identified and

Correspondence: Mitsuo Tagaya (tagaya@toyaku.ac.jp)

${ }^{1}$ School of Life Sciences, Tokyo University of Pharmacy and Life Sciences, Hachioji, Tokyo 192-0392, Japan

${ }^{2}$ Division of Regenerative Medicine, Jikei University School of Medicine, 3-25-8 Nishi-Shimbashi, Minato-ku, Tokyo 105-8461, Japan

Full list of author information is available at the end of the article.

Deceased: Katsuko Tani

Edited by: M. Diederich numbered (spastic paraplegia (SPG) 1-79). HSP-causing genes encode proteins involved in axon pathfinding, cytoskeleton organization, membrane trafficking, endoplasmic reticulum (ER) shaping, and mitochondrial functions ${ }^{1,2}$.

The intracellular phospholipase $\mathrm{A}_{1}\left(\mathrm{PLA}_{1}\right)$ protein family is a relatively recently discovered lipid-metabolizing enzyme family, and characterized by the presence of the short lipase active-site sequence Gly-X-Ser-X-Gly (X represents any amino acid) and a C-terminal DDHD (named after the presence of conserved three Asp residues and one His residue) domain. This family in mammals consists of three members ${ }^{4}$ : phosphatidic acid-preferring $\mathrm{PLA}_{1} / \mathrm{DDHD}^{5}$, p125/Sec23IP ${ }^{6}$, and KIAA0725p/ DDHD2 ${ }^{7}$. DDHD1 is highly expressed in brain and

\section{(c) The Author(s) 2018}

(c) (i) Open Access This article is licensed under a Creative Commons Attribution 4.0 International License, which permits use, sharing, adaptation, distribution and reproduction in any medium or format, as long as you give appropriate credit to the original author(s) and the source, provide a link to the Creative Commons license, and indicate if changes were made. The images or other third party material in this article are included in the article's Creative Commons license, unless indicated otherwise in a credit line to the material. If material is not included in the article's Creative Commons license and your intended use is not permitted by statutory regulation or exceeds the permitted use, you will need to obtain permission directly from the copyright holder. To view a copy of this license, visit http://creativecommons.org/licenses/by/4.0/. 
testis $^{5,8}$, and is involved in sperm formation ${ }^{9}$. Mutations in the $D D H D 1$ gene have been reported to cause $\mathrm{HSP}^{10}$, but no obvious SPG symptoms were observed in DDHD1 knockout (KO) mice'. Sec23IP is localized in ER exit sites $^{11}$, and the $\mathrm{KO}$ mice also exhibit a deficiency in spermiogenesis $^{12}$. Mutations in the DDHD2 gene also cause $\mathrm{HSP}^{13-17}$. Although DDHD1 is cytosolic ${ }^{8,9}$, DDHD2 is localized in both the cytosol and membranes including the Golgi apparatus ${ }^{7,18}$, and perhaps the ER ${ }^{7}$. For membrane binding, both lipase activity ${ }^{19}$ and a sterile alpha motif (SAM) domain flanked by the DDHD domain ${ }^{20}$ are important. Interestingly, treatment with CI-976, a lysophospholipid acyltransferase antagonist, causes the redistribution of cytosolic DDHD2 to tubular structures in close vicinity to mitochondria ${ }^{21}$.

Patients with $D D H D 2$ mutations are characterized by a thin corpus callosum ${ }^{13-17}$ and lipid accumulation in the brain, as detected on cerebral magnetic resonance spectroscopy ${ }^{13,17}$. The HSP phenotype and lipid accumulation were observed in DDHD2 KO mice ${ }^{22}$. Mass spectrometrybased lipidomics revealed that DDHD2 regulates brain triacylglycerol (TAG) levels, and that recombinant DDHD2 displayed TAG lipase activity ${ }^{22}$. Other studies demonstrated that DDHD2 exhibits diacylglycerol lipase activity $^{23,24}$. Although lipid droplet (LD) accumulation in the brain of DDHD2 KO mice is likely due to the lack of lipase activity derived from DDHD2, the reason why agedependent motor neuron degeneration occurs in SPG remains unknown. Here we show that DDHD2 ablation induces reactive oxygen species (ROS) production in mitochondria, thereby leading to apoptosis. Expression of wild-type (WT) DDHD2, but not the active-site mutant or mutants related to SPG, in DDHD2-deficient cells prevents ROS production and facilitates their consumption.

\section{Results \\ Loss of motor neurons in the spinal cords of DDHD2 KO mice}

To reveal the physiological function of DDHD2, we generated $D D H D 2 \mathrm{KO}$ mice. Using a targeting vector that contains exons 8 and 9 flanked by two loxP sites (Supplementary Figure 1a), we obtained targeted cell lines, and then generated chimeric, flox, and heterozygous and homozygous $\mathrm{KO}$ mice, as described under Materials and methods. Southern and Western blotting (WB) demonstrated the loss of exons 8 and 9 in the DDHD2 gene and DDHD2 protein, respectively, in the obtained mice (Supplementary Figure 1b). DDHD2 KO mice exhibited a paw clasping response (Supplementary Figure 1c) and reduced hind limb extension behavior (Supplementary Figure 1d) in an age-dependent manner. Moreover, reduced stride lengths were observed in 6month-old KO mice (Supplementary Figure 1e). These are typical features of $\mathrm{SPG}^{1,2}$.
Hematoxylin and eosin staining of the lumbar spinal cords of DDHD2 $\mathrm{KO}$ mice revealed the loss of motor neurons at 6 months, but not 1 month, of age (Fig. 1a). However, vacuoles were seen even in 1-month-old mice (Fig. 1a). Sudan III staining showed the accumulation of neutral lipids in the spinal cords of juvenile DDHD2 KO mice, but not of WT mice (data not shown), suggesting that LDs start to accumulate in juvenile DDHD2 KO mice. Immunostaining and WB demonstrated a marked reduction in cells positive for SMI32, a motor neuron marker ${ }^{25}$, in the lumbar spinal cords of 6-month-old DDHD2 KO mice (Fig. 1b, c). It should be noted that there was no substantial difference in the number of SMI32-positive cells between WT and DDHD2 KO mice at the age of 1 month. Concomitantly with the loss of motor neurons, the number of astrocytes positive for GFAP $^{26}$ increased (alternatively, astrocytes were activated and their size increased) (Fig. 1d). Consistent with the reduction of motor neurons, many apoptotic cells visualized as the formation of cleaved caspase 3 were observed in the spinal cords of DDHD2 $\mathrm{KO}$ mice at the age of 6 months, but not 1 month (Fig. 1e).

We investigated the viability of motor neurons in DDHD2 KO mice embryos. Neurons were dissected from the spinal cords and brains of embryos and then cultured. At day 2, most spinal cord motor neurons from $D D H D 2$ KO mice had died, although those from WT mice survived well (Fig. 1f). On the other hand, neurons in the motor cortex of DDHD2 KO mice survived, similar to those from the WT mice (Supplementary Figure 1f). These results suggest that the DDHD2 deficiency causes degeneration of motor neurons in the spinal cord, not the motor cortex, in an age-dependent manner.

\section{Loss of DDHD2 increased sensitivity to apoptosis in vivo and in vitro}

Given that motor neurons in the spinal cords of $D D H D 2 \mathrm{KO}$ mice not only underwent apoptosis in an age-dependent manner, but also could not survive in vitro even at the embryonic stage, we reasoned that motor neurons of $D D H D 2 \mathrm{KO}$ mice might suffer from some stresses due to DDHD2 ablation. Moreover, such stresses might occur not only for motor neurons but also for other cells. Indeed, mouse embryo fibroblasts (MEFs) from DDHD2 KO mice could not proliferate well (data not shown). We therefore generated immortalized MEFs by introducing large $\mathrm{T}$ antigen using a retrovirus expression system. Unless otherwise described, we used immortalized MEFs for further studies. We first confirmed that DDHD2 protein was expressed in WT MEFs, but not DDHD2 KO MEFs (Fig. 2a). Next, we examined the sensitivity of MEFs to staurosporine (STS), a well-known apoptosis inducer that preferentially activates $\mathrm{Bax}^{27}$. On treatment with STS, the percentage of TUNEL-positive cells increased more 
a
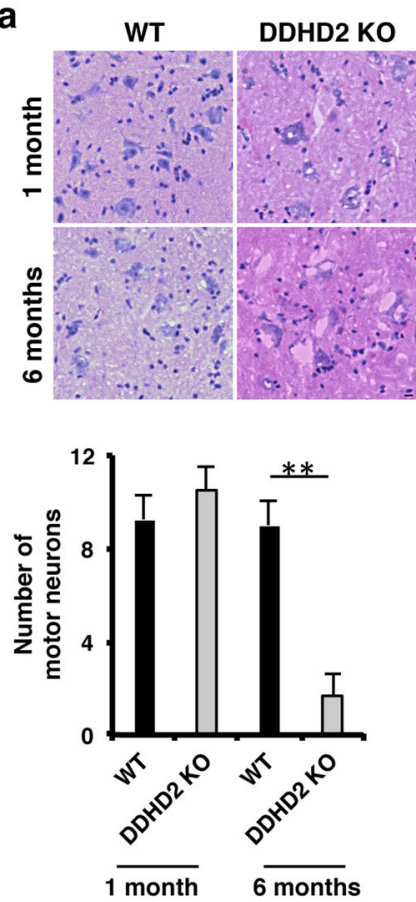

d
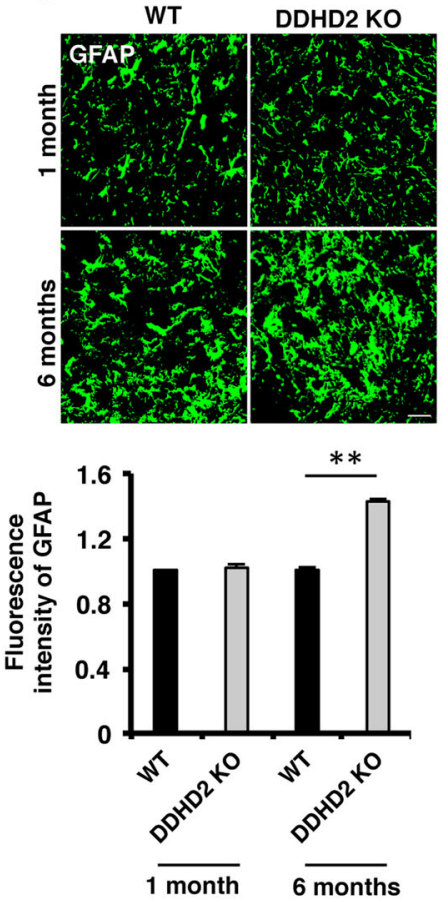

b
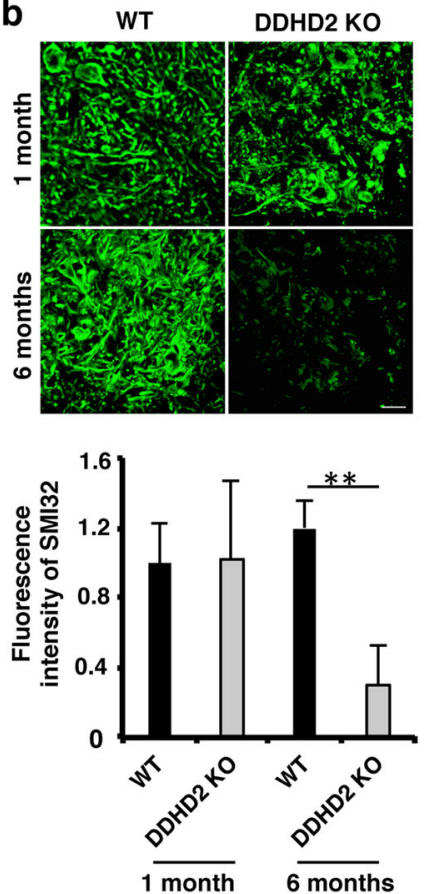

e
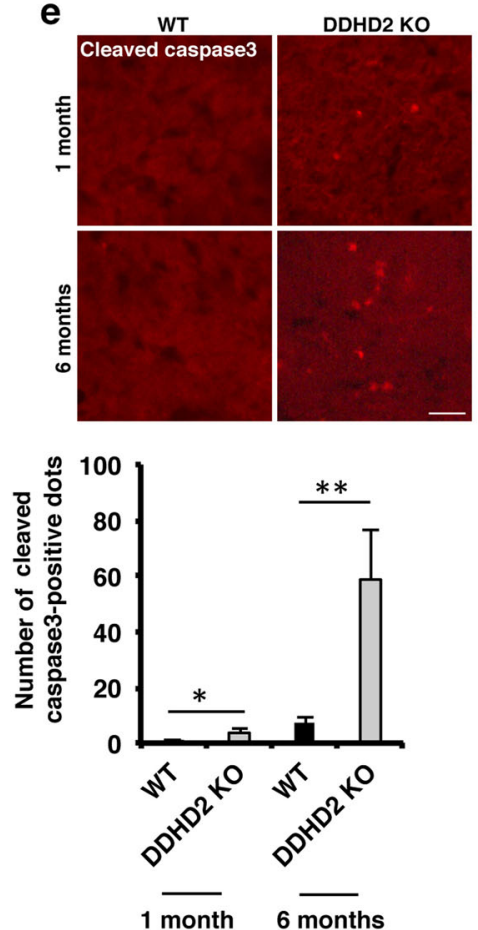

C
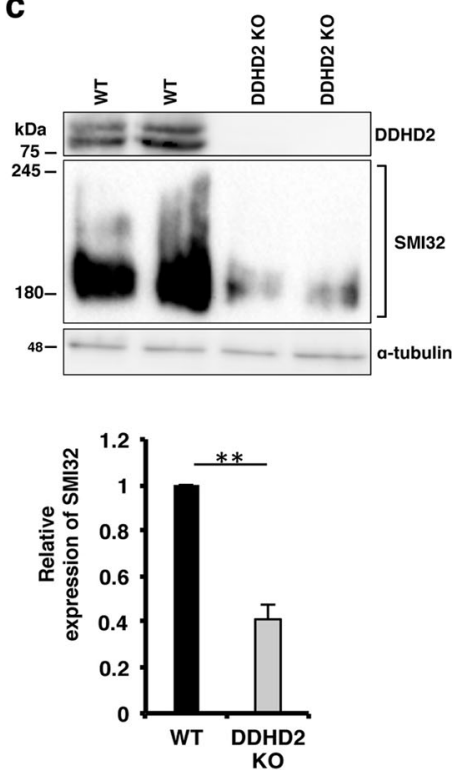

f
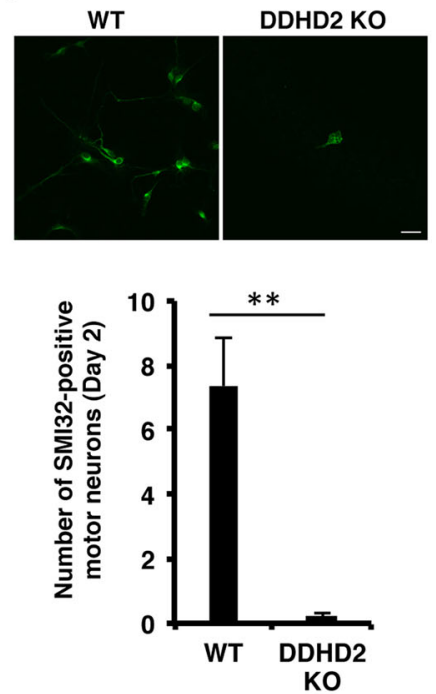

Fig. 1 Loss of DDHD2 induces motor neuron degeneration in the spinal cord. a Hematoxylin and eosin staining of lumbar spinal cords of WT and DDHD2 KO mice The motor neurons were counted. Values are means \pm SEM $(N=3)$. $\mathbf{b}$, $\mathbf{d}$, e Photomicrographs of cross-sections of the lumbar spinal cords of WT and DDHD2 KO mice labeled with an antibody against SMI32 (b), GFAP (d), or cleaved caspase3 (e). The total fluorescence intensity or the total number of cleaved caspase3-positive cells in each spinal cord section was determined. Six sections were analyzed in each mouse, and three mice were used for each experiment. Data represent means \pm SEM $(N=3)$. c Spinal cord lysates $(20 \mu \mathrm{g})$ of WT and DDHD2 KO mice at 6 months of age were analyzed by WB with antibodies against DDHD2, SMI32, and a-tubulin. The intensity of immunoreactive signals was quantified, and the SMI32 level in KO mice relative to that in $\mathrm{WT}$ mice is expressed as means \pm SEM $(N=3)$. f Primary motor neurons from the spinal cords of WT and DDHD2 KO mice were cultured for 2 days, and then immunostained with an anti-SMI32 antibody. The number of surviving SMI32-positive motor neurons in an arbitrarily chosen microscopic field with a size of $212 \times 212 \mu \mathrm{m}^{2}$ was determined. Six fields were analyzed in each experiment. Data represent means \pm SEM $(N=3)$. The scale bars for whole cell panels are $10 \mu \mathrm{m} .{ }^{*} P<0.05,{ }^{* *} P<0.01$, Student's $t$-test 

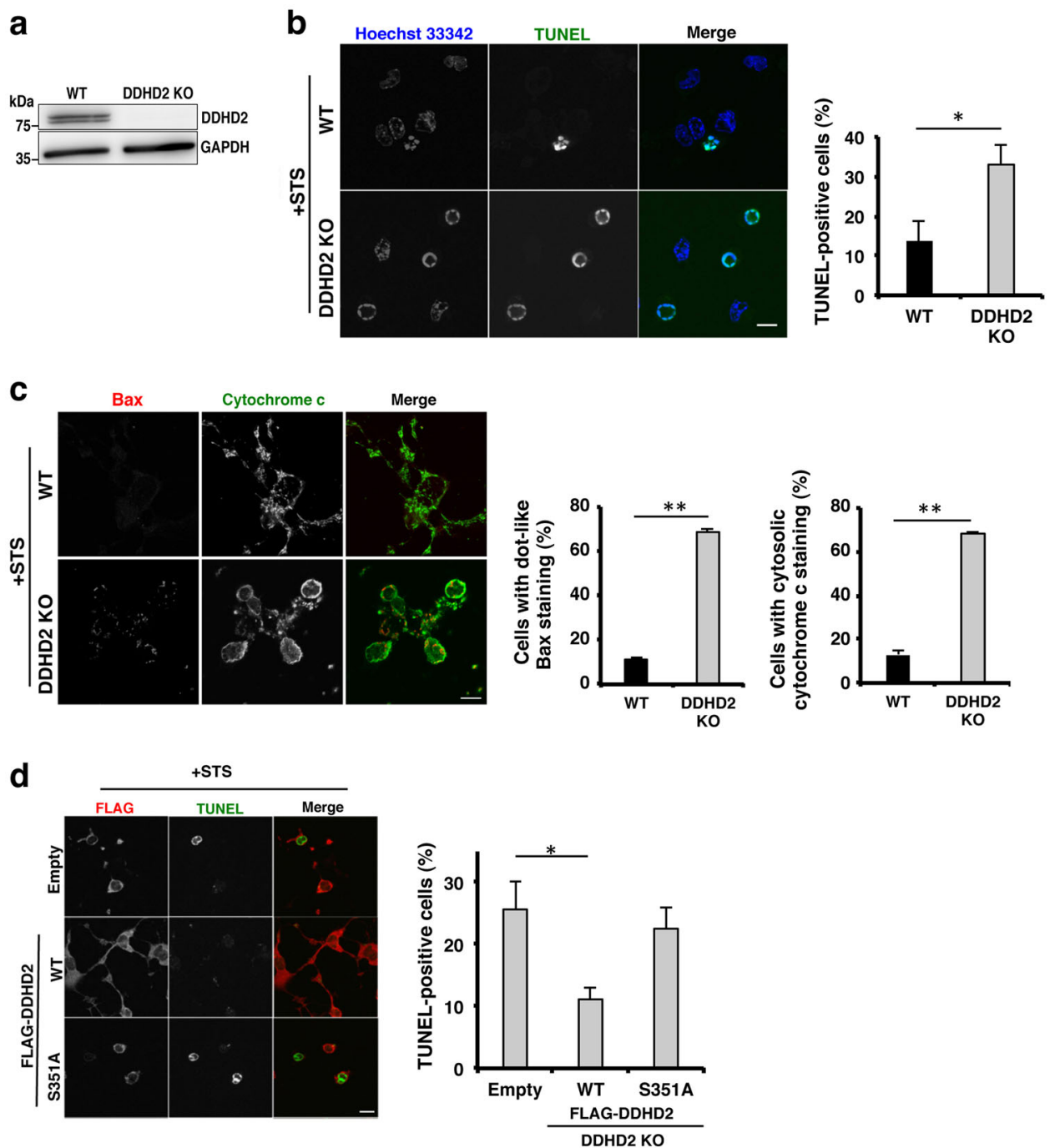

Fig. 2 Loss of DDHD2 increases apoptosis sensitivity of MEFs. a Lysates $(10 \mu \mathrm{g})$ of WT and DDHD2 KO MEFs were analyzed by WB with antibodies against DDHD2 and GAPDH. b, c WT and DDHD2 KO MEFs were treated with $1 \mu \mathrm{M}$ STS for 8 h, followed by staining with TUNEL and Hoechst 33342 (b), or antibodies against Bax and cytochrome $\mathrm{c}$ (c). The number of TUNEL-positive cells or cells exhibiting dot-like Bax staining or cytosolic cytochrome c staining was determined. d DDHD2 KO MEFs were transfected with a plasmid encoding FLAG (Empty), FLAG-DDHD2 WT, or FLAGDDHD2-S351A. At $24 \mathrm{~h}$ after transfection, STS was added to a final concentration of $1 \mu \mathrm{M}$, and the cells were incubated for $8 \mathrm{~h}$, and then subjected to staining with TUNEL and an anti-FLAG antibody. The number of TUNEL-positive cells was determined. No significant difference in the percentage of TUNEL-positive cells was observed between cells transfected with FLAG and FLAG-DDHD2-S351A. Data represent means \pm SEM for nine (b) and three (c, d) independent experiments, respectively. The scale bars for whole cell panels are $10 \mu \mathrm{m} .{ }^{*} P<0.05,{ }^{* *} P<0.01$, Student's $t$-test

than two-fold in comparison to WT cells (Fig. 2b). Consistent with the results of the TUNEL analysis, the percentages of cells with dot-like staining for the proapoptotic protein Bax and cytosolic staining for cytochrome c were much higher in DDHD2 KO MEFs than WT cells (Fig. 2c). Importantly, the enhancement of STSinduced apoptosis in DDHD2 KO MEFs was reversed by the expression of FLAG-DDHD2 WT, but not the S351A mutant, in which active-site residue Ser351 was replaced by Ala (Fig. 2d), suggesting that the enzymatic activity of DDHD2 is important for cell survival. To determine whether the enhanced sensitivity to apoptosis is not limited to MEFs, we used U2OS cells. We knocked down DDHD2 in U2OS cells with two short interfering RNAs (siRNAs), and then examined the sensitivity of DDHD2depleted cells to STS and $\mathrm{H}_{2} \mathrm{O}_{2}$. Upon knockdown of DDHD2, the percentages of TUNEL-positive cells and cleaved caspase3-positive cells in response to STS and $\mathrm{H}_{2} \mathrm{O}_{2}$ treatment, respectively, substantially increased (Supplementary Figures 2a and b). These results suggest that loss of DDHD2 causes stresses not only for motor neurons but also for cells in general. 


\section{Accumulation of ROS in DDHD2 KO and silenced cells}

Increased levels of ROS are known to cause damage to lipids, proteins, and DNA, often leading to cell death. Motor neurons are quite vulnerable to oxidative stress, as evidenced by the fact that mutations in superoxide dismutase 1, an enzyme that consumes superoxide radicals, induces amyotrophic lateral sclerosis ${ }^{28}$. Therefore, we next examined whether loss of DDHD2 increased the level of intracellular ROS. We used CellROX, a fluorogenic probe that can detect ROS in both live and fixed cells. We first examined MEFs without immortalization treatment. The intensity of CellROX fluorescence was three-fold higher in DDHD2 KO MEFs than WT MEFs (Fig. 3a). In the case of immortalized MEFs, the intensity of CellROX fluorescence in DDHD2 KO MEFs was 25\% higher than that in WT MEFs (Fig. 3b). However, the expression levels of enzymes responsible for the clearance of ROS, such as superoxide dismutase 1 and catalase were not significantly affected (Fig. 3c). ROS production was also detected in siRNA-silenced U2OS cells (Supplementary Figure 3a). It should be noted that the stable expression of DDHD2-WT-mCherry in DDHD2 $\mathrm{KO}$ MEFs caused a $62 \%$ reduction in the intensity of CellROX staining, whereas no reduction was observed when the S351A mutant was expressed (Fig. 3b). Expression of the FLAG-DDHD2 mutants related to SPG also failed to decrease the intensity of CellROX staining (Fig. 3d). Moreover, stable expression of DDHD1, another member of the intracellular $\mathrm{PLA}_{1}$ family, could not reverse the enhanced CellROX staining (Supplementary Figure 4), suggesting that the two family members play different roles.

\section{Mitochondrial ROS production in DDHD2 KO cells impairs mitochondrial function}

Because the major source of ROS in cells is mitochondria, we examined whether ROS formation in the absence of DDHD2 occurs at mitochondria. DDHD2 KO MEFs were double stained with CellROX and mitochondrial proteins (Tom20 and cytochrome c). The CellROXpositive structures almost completely overlapped with the mitochondrial proteins (Fig. 4a). We used MitoSOX, a probe that specifically detects ROS produced in mitochondria. The MitoSOX staining also overlapped with the MitoTracker Green FM staining (Fig. 4b). In DDHD2 KO MEFs, the ability of ATP generation decreased (Fig. 4c). In parallel with this, the mitochondrial membrane potential estimated as the ratio of green to red fluorescence of JC-1 increased (Fig. 4d), and the oxygen consumption rate (OCR) decreased (Fig. 4e). In U2OS cells, the intensity of MitoSOX staining increased upon DDHD2 depletion (Supplementary Figure 3b). Consistent with the view that ROS is responsible for apoptosis, incubation of U2OS cells depleted of DDHD2 with $N$-acetylcysteine (NAC) prevented apoptosis, as visualized as prevention of caspase3 cleavage (Supplementary Figure 3c).

We next examined whether DDHD2 can protect cells from oxidative stress. To this end, we used antimycin Arotenone (AR) and paraquat (PQ) to produce ROS in mitochondria. First, we confirmed that DDHD2 KO MEFs were more susceptible to AR and PQ than WT MEFs (Fig. 5a), and that this susceptibility was reversed by expression of DDHD2-WT-mCherry, but not the S351A mutant (Fig. 5b). ROS generation induced by PQ was suppressed by the expression of the FLAG-DDHD2 WT, but not the S351A mutant or the mutants related to SPG, in WT MEFs (Fig. 5c). These results suggest that DDHD2 is responsible for the prevention of ROS generation.

Next, we examined whether DDHD2 contributes to the removal of formed ROS. DDHD2 KO MEFs and WT MEFs were incubated with AR for $3 \mathrm{~h}$ to allow ROS accumulation, followed by incubation in the absence of AR to monitor ROS consumption. In WT MEFs, the CellROX staining was decreased by $66 \%$ at $3 \mathrm{~h}$ after AR washout, whereas only $18 \%$ reduction was observed for DDHD2 KO MEFs (Fig. 5d), suggesting that DDHD2 contributes to both the prevention of ROS formation and removal of formed ROS.

\section{LDs are not accumulated in DDHD2 KO MEFs}

A previous study demonstrated the accumulation of LDs in the brain of DDHD KO mice ${ }^{22}$. We therefore examined whether DDHD2 KO MEFs also contain increased levels of LDs and TAG. However, LipiDye staining showed that the numbers of LDs were not significantly different between WT and DDHD2 KO MEFs (Supplementary Figure 5a). Similarly, no significant difference in the number of LDs was observed between WT and DDHD2 KO MEFs when LD formation was induced by oleic acid (OA). The TAG content was also indistinguishable between WT and DDHD2 KO MEFs regardless of whether $\mathrm{LD}$ formation was induced by $\mathrm{OA}$ or not (Supplementary Figure 5b). These results indicate that the formation of ROS in DDHD2 KO MEFs is not due to the accumulation of neutral lipids.

\section{Reaction of lipid peroxidation sensors in DDHD2 MEFs}

ROS formation causes lipid peroxidation ${ }^{29}$. We monitored possible lipid peroxidation using two fluorescence sensors, MitoPeDPP ${ }^{30}$ and BODIPY 581/591 C11 ${ }^{31}$. Both sensors detected lipid peroxidation in DDHD2 KO MEFs (Supplementary Figures $6 \mathrm{a}$ and b). The increased MitoPeDPP staining was suppressed by the expression of DDHD2-WT-mCherry, but not the S351A mutant (Supplementary Figure 6c). To determine whether possibly oxidized lipids can be removed by DDHD2, WT and DDHD2 KO MEFs were incubated with $1 \mathrm{mM}$ tert-butyl 


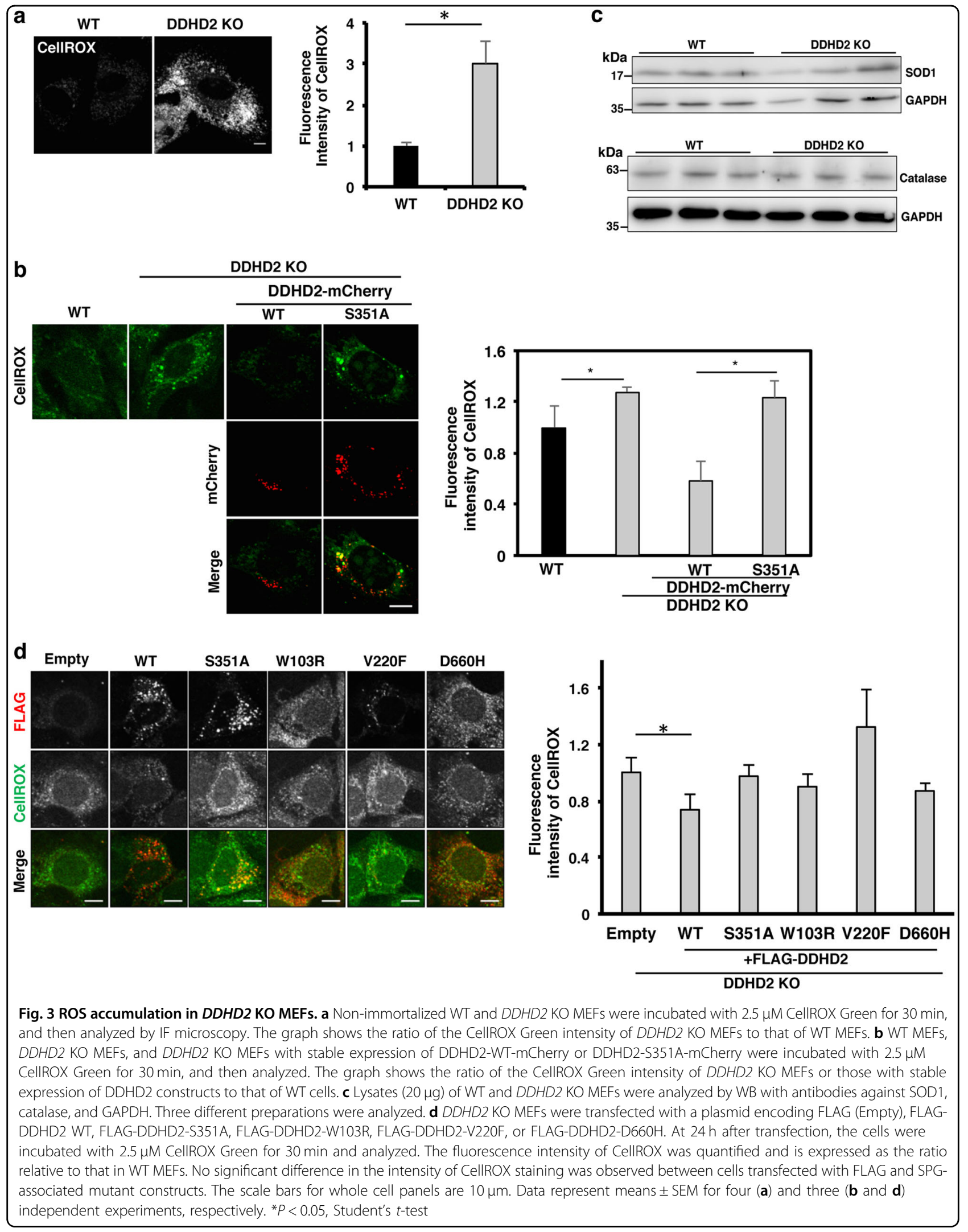




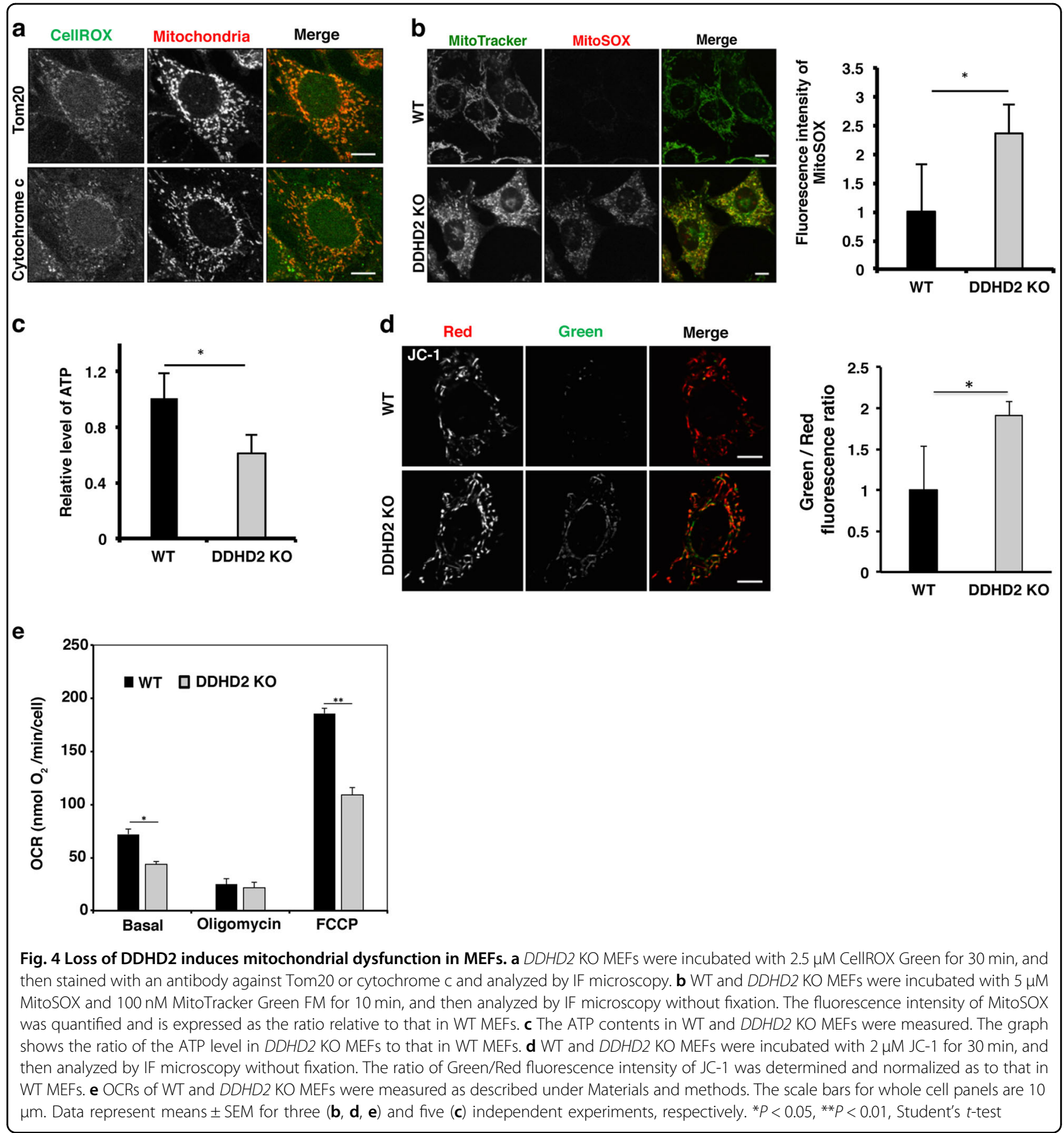

hydroperoxide (t-BHP) for $30 \mathrm{~min}$ to allow the accumulation of oxidized lipids, followed by incubation in the absence of t-BHP to monitor degradation of the oxidized lipids formed. In WT MEFs, BODIPY 581/591 C11 green fluorescence reflecting possible lipid peroxidation had decreased by $58 \%$ at $1 \mathrm{~h}$ after t-BHP washout, whereas no substantial decrease in green fluorescence was observed in DDHD2 KO MEFs, suggesting that DDHD2 can cleave possibly oxidized lipids (Supplementary Figure 6d; Supplementary Figure 7).

When lipid peroxidation occurs, 4-hydroxynonenal (4-HNE) and malondialdehyde (MDA) are formed as degradation products of oxidized lipids ${ }^{29}$. We therefore examined by WB lysates of spinal cords of 6-month-old DDHD2 KO mice and detected 4-HNEmodified proteins (Supplementary Figure 6e). Similarly, 
a

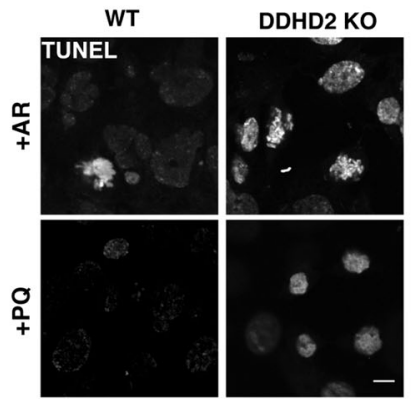

b

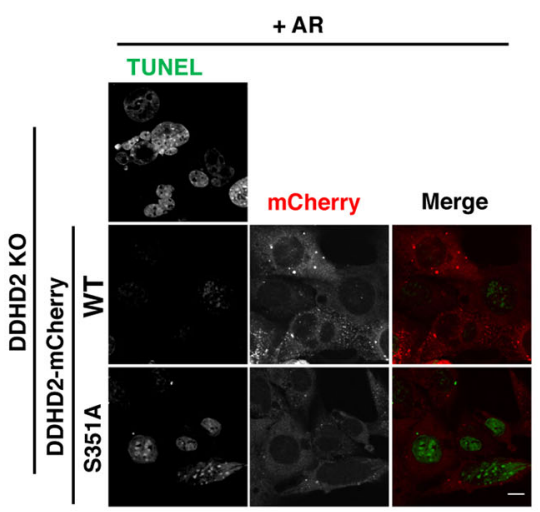

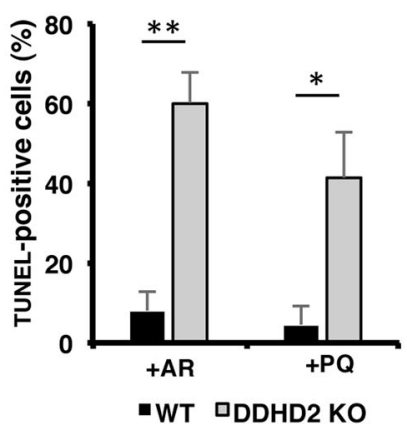

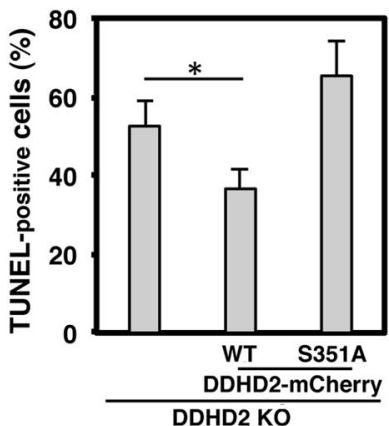

C

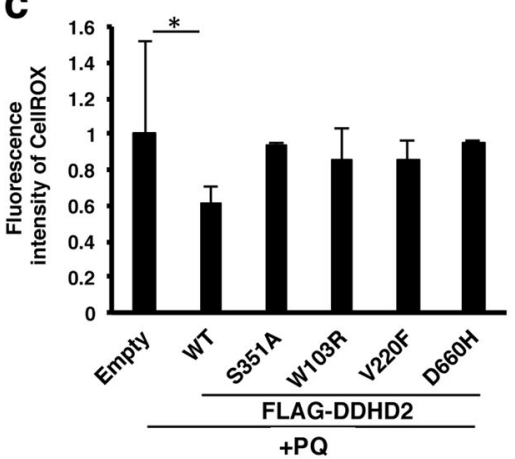

d

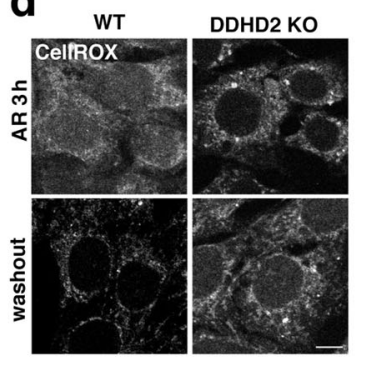

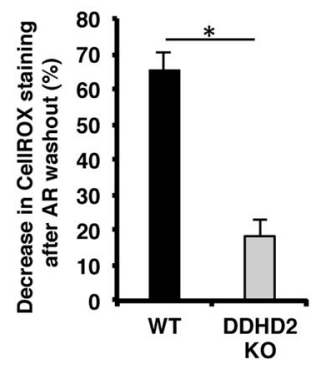

Fig. 5 Loss of DDHD2 increases ROS-induced apoptosis sensitivity and delays ROS clearance in MEFs. a WT and DDHD2 KO MEFs were treated with $2 \mu \mathrm{M}$ antimycin $\mathrm{A}$ and $1 \mu \mathrm{M}$ rotenone (AR) or $0.1 \mathrm{mM} \mathrm{PQ}$ for $16 \mathrm{~h}$, followed by staining with TUNEL. The number of TUNEL-positive cells was determined. $\mathbf{b}$ DDHD2 KO MEFs and ones with stable expression of DDHD2-WT-mCherry or DDHD2-S351A-mCherry were treated with AR for $16 \mathrm{~h}$, followed by staining with TUNEL. The number of TUNEL-positive cells was determined. $\mathbf{c}$ WT MEFs were transfected with a plasmid encoding FLAG (Empty), FLAG-DDHD2 WT or one of the indicated constructs. At $24 \mathrm{~h}$ after transfection, the cells were treated with $0.1 \mathrm{mM} P Q$ for $1 \mathrm{~h}$, followed by incubation with $2.5 \mu \mathrm{M}$ CellROX Green for $30 \mathrm{~min}$ and analysis. The fluorescence intensity of CellROX Green was quantified and is expressed as the ratio relative to that in MEFs with FLAG vector transfection. No significant difference in the intensity of CellROX staining was observed between cells transfected with FLAG and SPG-associated mutant constructs. $\mathbf{d}$ WT and DDHD2 KO MEFs were incubated with $2 \mu \mathrm{M}$ antimycin A and $1 \mu \mathrm{M}$ rotenone (AR) for $3 \mathrm{~h}$, washed, and then incubated without AR for $1 \mathrm{~h}$. To detect ROS, cells were incubated with $2.5 \mu \mathrm{M}$ CellROX Green for 30 min. The fluorescence intensity of CellROX Green relative to that observed just after 3-h AR treatment was determined. The scale bars for whole cell panels are $10 \mu \mathrm{m}$. Data in this figure represent means $\pm \operatorname{SEM}(N=3) .{ }^{*} P<0.05,{ }^{* *} P<0.01$, Student's $t$-test

4-HNE-modified proteins were detected in lysates of DDHD2 KO MEFs (Supplementary Figure 6f). Moreover, an enhanced level of MDA was observed in brain lysates of 6-month-old DDHD2 KO mice (Supplementary Figure 6g).

\section{Decreased cardiolipin (CL) levels in DDHD2 KO MEFs}

When MEFs were subjected to lipid analysis by mass spectrometry, unexpectedly we did not detect oxidized phospholipids but found a substantial decrease in CL content (Fig. 6a). Some other phospholipids including 

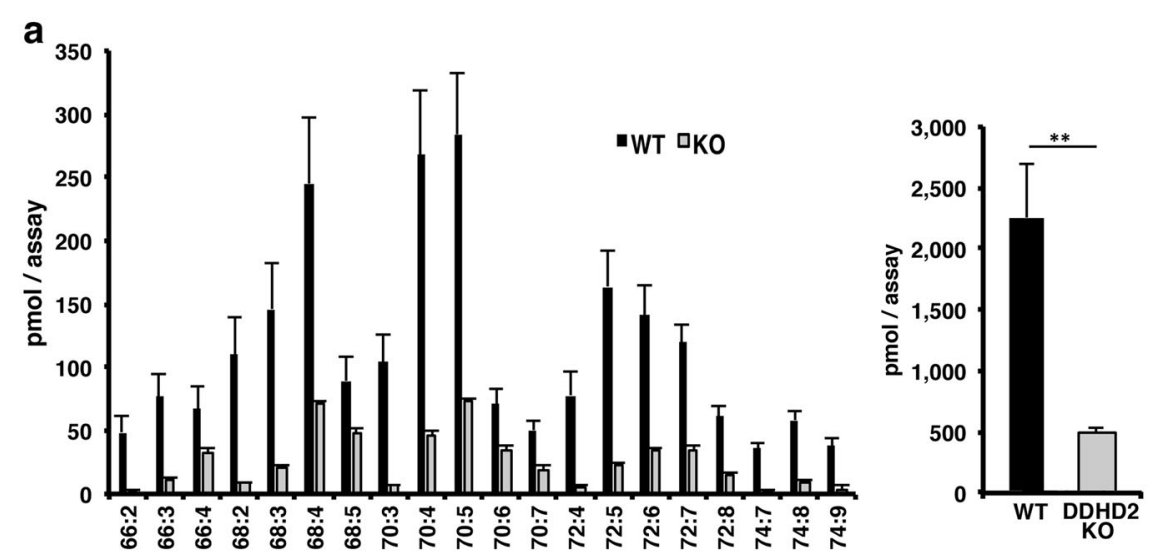

b
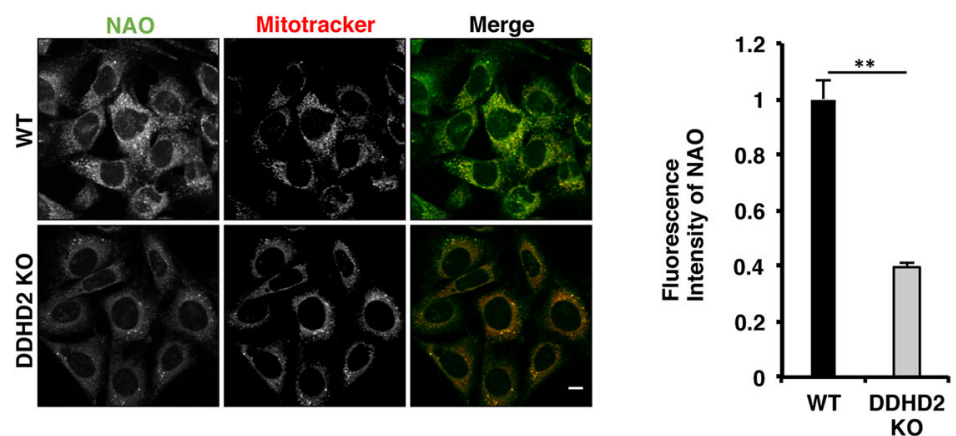

Fig. 6 Reduction of CLs in DDHD2 KO MEFs. a The CL species in WT and DDHD2 KO MEFs were analyzed by mass spectrometry, as described under Materials and methods. The bar graph shows the total amounts of CLs. b WT and DDHD2 KO MEFs were incubated with $1 \mu \mathrm{M}$ NAO and $500 \mathrm{nM}$ MitoTracker Red FM for $30 \mathrm{~min}$, and then analyzed by IF microscopy. The scale bar is $10 \mu \mathrm{m}$. The fluorescence intensity of NAO was quantified and is expressed as the ratio relative to that of WT MEFs. Data in this figure represent means $\pm \operatorname{SEM}(N=3)$. ${ }^{*} P<0.01$, Student's $t$-test

phosphatidylcholine appeared to be slightly decreased, but not significant (data not shown). Consistent with the results of mass spectrometric analysis, the staining intensity of $D D H D 2 \mathrm{KO}$ MEFs with $10-N$-nonyl acridine orange (NAO), a CL-specific probe commonly used to monitor CL levels in live cells ${ }^{32}$, was lower than that of WT MEFs (Fig. 6b).

\section{DDHD2 localizes to ROS-producing mitochondria}

Our previous study demonstrated that DDHD2 localizes to the ER, Golgi apparatus, and cytosol ${ }^{7,19,20}$. Upon treatment of cells with CI-976, a lysophospholipid acyltransferase antagonist, DDHD2 was found to be redistributed to tubular structures in close vicinity to mitochondria $^{21}$. Given that DDHD2 contributes to the removal of mitochondrial ROS and oxidized lipids, we explored the possibility that DDHD2 interacts with mitochondria. On immunofluorescence (IF) microscopy, DDHD2 in MEFs exhibits a dispersed pattern (Fig. 7a), and this staining was abolished upon DDHD2 KO (data not shown), suggesting the specificity of the antibody used. When MEFs were incubated with reagents that cause oxidative stress, DDHD2 formed large aggregates
(Fig. 7a, right, top, second, and third rows), whereas such aggregation was not obvious upon treatment with tunicamycin, an ER stress inducer (bottom row).

We performed live cell imaging using DDHD2-WTmCherry and the S351 mutant expressed in DDHD2 KO MEFs. DDHD2-WT-mCherry moved and transiently stayed together with MitoPeDPP (Fig. 7b; Supplementary Movie 1), whereas the S351 mutant appeared to consistently associate with MitoPeDPP with much less movement (Fig. 7c; Supplementary Movie 2). These results suggest that DDHD2 can interact with mitochondria, and that its inactive mutant stays longer compared with WT DDHD2.

\section{Discussion}

In the present study we demonstrated that not only motor neurons but also MEFs from DDHD2 $\mathrm{KO}$ mice cannot proliferate well in vitro. We found that both DDHD2 KO MEFs and U2OS cells depleted of DDHD2 by siRNAs are susceptible to apoptotic stimuli. Further analysis revealed that loss of DDHD2 causes ROS production in mitochondria. The notion that ROS production is responsible for the enhanced apoptosis 


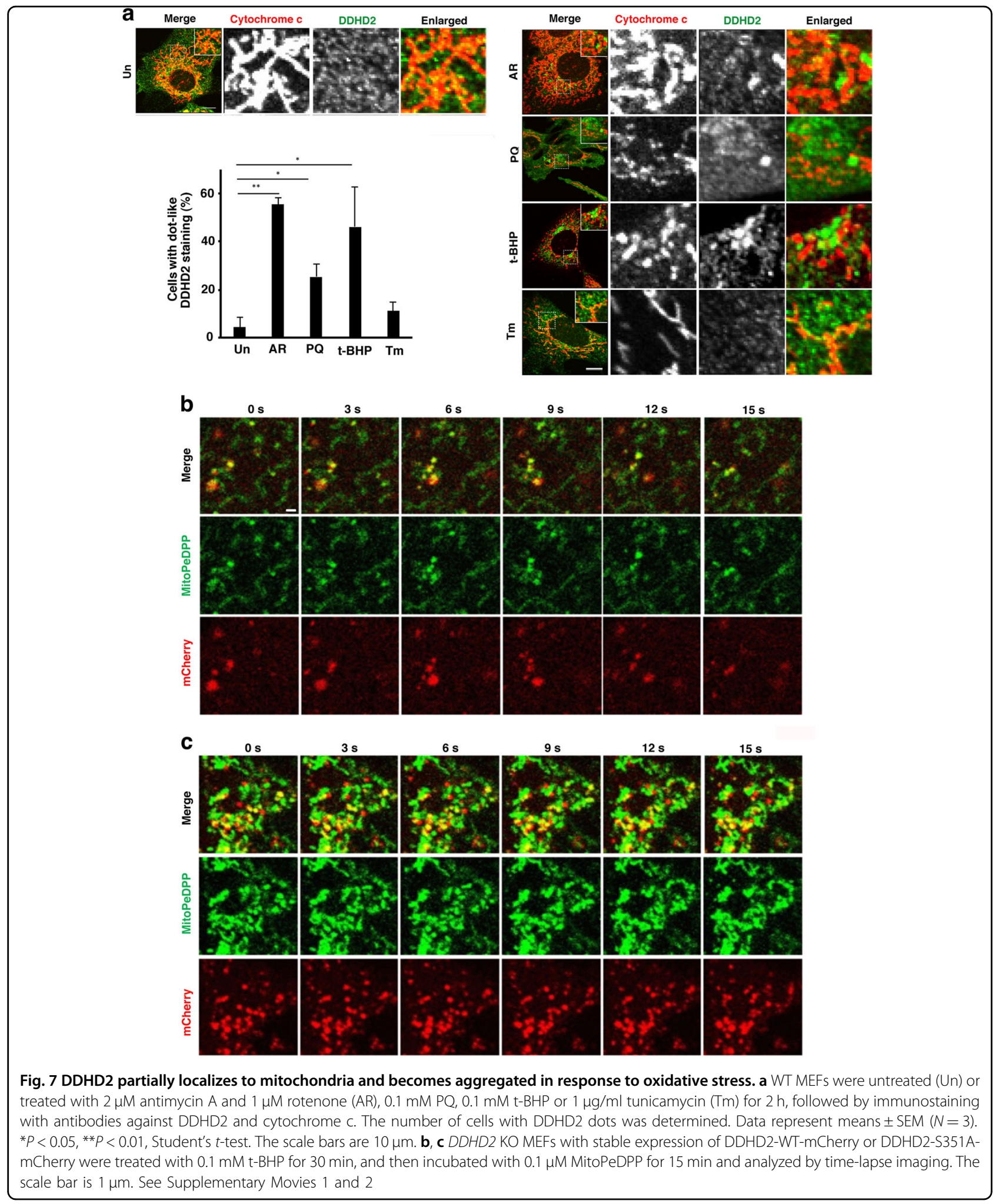

sensitivity was corroborated by the finding that NAC prevented apoptosis. Enhanced generation of ROS upon DDHD2 ablation might produce lipid electrophiles, which interfere with protein function through modification, leading to apoptosis of cultured cells and perhaps motor neurons. Expression of WT DDHD2 in DDHD2-depleted 
cells not only prevented the formation of ROS, but also facilitated its clearance. It is of note that DDHD2 deficiency could not be compensated for by the expression DDHD2 mutants related to SPG, as well as the active-site mutant. In addition, DDHD2 could not be substituted with DDHD1, another member of the PLA 1 family implicated in SPG28.

Recent studies revealed that ROS production and LD accumulation are closely correlated ${ }^{33}$. In hepatocytes, ROS levels were found to be proportional to LD accumulation $^{34}$. In Drosophila, LDs formed in glial cells during oxidative stress accommodate polyunsaturated fatty acids that have been present in the plasma membrane before oxidative stress, which possibly protects polyunsaturated fatty acids from lipid peroxidation by keeping them away from the ROS-sensitive plasma membrane pool ${ }^{35}$. In the case of photoreceptors (neurons), ROS production stimulates the synthesis of lipids in neurons that are subsequently delivered to neighboring glial cells to induce LD accumulation $^{36}$. A subsequent study by the same group suggested that LD accumulation prevents neurodegeneration through scavenging of peroxidized lipids ${ }^{37}$. In DDHD2 KO mice, LDs were found to accumulate in the brain (ref. ${ }^{22}$ and this study). This can be explained by the finding that DDHD2 has TAG lipase activity ${ }^{22}$. However, lack of the TAG lipase activity in the brain may not be a sole reason for LD accumulation. Our present results demonstrated that DDHD2-depleted cells generate ROS, raising the possibility that $L D$ accumulation in the brain of DDHD2 KO mice is partly a consequence of ROS generation. MEFs may express higher levels of TAG lipases than neuronal cells, thereby preventing LD accumulation, even ROS generation. Taken together, ROS generation, as well as LD accumulation, may contribute to the pathogenesis of SPG.

Mass spectrometric analysis revealed that the CL levels are decreased in DDHD2 KO MEFs. This is in contrast to the case of yeast homolog Ddl1 (Yor022c); loss of the Ddl1 gene resulted in an increase $(\sim 21 \%)$ in the CL content $^{38}$. Yadav and colleagues ${ }^{38}$ hypothesized that yeast $\mathrm{PLA}_{1}$ Ddl1, in cooperation with phospholipase $\mathrm{A}_{2}$ Cld1, participates in CL remodeling, and that dilysocardiolipin formed by a sequential cleave by the two enzymes undergo degradation. The reason for these opposite results between yeast and mammals is not known at present, but the present results raise the possibility that DDHD2 also directly or indirectly participates in CL remodeling. CLs play important roles for mitochondrial functions. They are essential for the architecture and activity of mitochondrial respiratory chain complexes ${ }^{39}$. Therefore, reduction of CLs in DDHD2 KO MEFs can explain the phenotype of cells: ROS production and reduction in ATP production, mitochondrial membrane potential, and respiratory activity.
Mass spectrometric analysis also revealed no marked accumulation of oxidized lipids in DDHD2 KO MEFs. This is rather surprising because the lipid peroxidationdegradation products 4-HNE and MDA seem to be significantly increased in DDHD2-depleted cells (Supplementary Figures $6 \mathrm{e}-\mathrm{g}$ ). Moreover, positive signals were seen for two lipid peroxidation sensors, MitoPeDPP and a commonly used BODIPY 581/591 C11 (Supplementary Figures $6 \mathrm{a}$ and $\mathrm{b}$ ). One explanation for these apparent inconsistencies is that ROS generation may be massive enough to oxidize lipid sensors, but not endogenous lipids. It has been reported that BODIPY 581/591 C11 is more sensitive to oxidative stress than authentic phospholipids ${ }^{40}$. Alternatively, but not exclusively, peroxidized lipids may promptly undergo nonenzymatic Hock cleavage, producing 4-HNE and MDA.

In conclusion, the present study revealed that DDHD2 plays a role in preventing ROS production and protecting cells against oxidative stress, although the mechanisms for these functions remain obscure. One possibility is that DDHD2 cleaves oxidized lipids to mitigate oxidative stress. This is consistent with the findings that the expression of DDHD2 can prevent the reaction of lipid peroxidation sensors and that DDHD2 forms aggregates upon oxidative stress and moves together with MitoPeDPP-positive mitochondria. In future studies the possibility that DDHD2 cleaves oxidized lipids should be tested. To develop drugs for SPG, DDHD2 KO mice may be useful. Obviously, antioxidants that block ROS formation are good candidates that may ameliorate the progression of HSP.

\section{Materials and methods \\ Animals}

All animal procedures and experiments were approved by the Animal Care Committee of Tokyo University of Pharmacy and Life Sciences, and conducted according to the guidelines of the committee.

\section{Plasmid construction}

The mammalian expression plasmids for FLAG-tagged human DDHD2 and its S351A mutant were described previously ${ }^{7}$. FLAG-tagged human DDHD2 SPG mutants were generous gifts from Dr. Hiroshi Doi (Yokohama City University).

\section{Retrovirus expression system}

The DNA fragments encoding pENTER-tagged human DDHD1, and DDHD2 and its mutants were inserted into the retrovirus vector pMRX-IRES-puro-DEST-mCherry. For the production of recombinant retroviruses, PLAT-E cells were transfected with retrovirus vectors using a PEI MAX reagent according to the manufacturer's protocol. To establish stable cell lines, cells were infected with 
recombinant retroviruses and cultured in medium containing $10 \mu \mathrm{g} / \mathrm{ml}$ puromycin.

\section{RNA interference}

The siRNA targeting sequences used were as follows: Luciferase siRNA, CGTACGCGGAATACTTCGA; DDH D2 siRNA\#2, AAGAAAGAAGAUAUUAAACUA; and DDHD2 siRNA\#3, AAGGAGAAAGUAGAUAAGGAA. U2OS cells were transfected with $100 \mathrm{nM}$ siRNA using Lipofectamine RNAiMAX (Thermo Fisher Scientific) according to the manufacturer's protocol. Cells were fixed and processed at $72 \mathrm{~h}$ after transfection.

\section{Cell culture and plasmid transfection}

MEFs and U2OS cells were grown in DMEM supplemented with $10 \%$ fetal calf serum (FCS). Plasmids were transfected using Lipofectamine 3000 (Thermo Fisher Scientific) or Neon (Thermo Fisher Scientific) according to the manufacturer's protocol.

\section{Preparation of MEFs and cell culture}

MEFs were isolated from E13.5 embryos and immortalized with the SV40 large T antigen. The immortalized cells were cultured in medium containing $2 \mu \mathrm{g} / \mathrm{ml}$ puromycin.

\section{Antibodies and probes}

A monoclonal antibody against 4-HNE (No. ab48506; 1:100 dilution for WB) and a polyclonal antibody against $\alpha$-tubulin (No. ab4074; 1:100,000 dilution for WB) were obtained from Abcam. A polyclonal antibody against SOD1 (No. HPA001401, 1:1000 for WB), monoclonal antibodies against GFAP (No. G3893; 1:200 dilution for IF), and FLAG (No. F7425; 1:1000 dilution for IF) were obtained from Sigma-Aldrich. A polyclonal antibody against Tom20 (No. FL-145; dilution for 1:1000), monoclonal antibodies against Bax (No. sc-493; 1:200 dilution for IF), and glyceraldehyde 3-phosphate dehydrogenase (GAPDH) (sc-47724; 1:1000 dilution for WB) were purchased from Santa Cruz Biotechnology. Monoclonal antibodies against SMI32 (No. 801701; 1:1000 dilution for IF and WB) and cytochrome c (No. 556432; 1:1000 dilution for IF) were purchased from BioLegend and $\mathrm{BD}$ Pharmingen, respectively. A polyclonal antibody against cleaved caspase3 (No. 9664; 1:1000 dilution for IF) was purchased from Cell Signaling. A polyclonal antibody against catalase (No. 219010, 1:1000) was purchased from Merck Millipore. A polyclonal antibody against mouse DDHD2 was raised against a recombinant GST-tagged DDHD2 fragment (amino acids 368-476) and affinitypurified using antigen-coupled beads. MitoTracker Green and Red CMXRos, MitoSOX Red Mitochondrial Superoxide Indicator, CellROX Green reagent, and BODIPY
581/591 C11 were purchased from Thermo Fisher Scientific. MitoPeDPP was purchased from Dojindo.

\section{Generation of DDHD2 knock-out mice}

A DDHD2 gene fragment was isolated from a mouse genomic bacterial artificial chromosome library derived from the 129sv mouse strain (RP22-60F20) and subcloned into pBluescript II SK. A conditional targeting vector was constructed using a highly efficient recombineering-based method $^{41}$. The DDHD2 targeting vector was digested with NotI, and then electroporated into E14 embryonic stem cells. Cells resistant to G418 were picked up and analyzed. Targeted cell lines were injected into C57BL/6J blastocysts to produce chimeric mice. The male chimeric mice were crossed with female WT C57BL/6J mice, by which DDHD2 ${ }^{+/ \text {flox }}$ offspring were produced. To disrupt the DDHD2 gene, DDHD2 ${ }^{+/ \text {flox }}$ offspring were mated with CAG-Cre transgenic mice (RIKEN BRC) ${ }^{42}$, which express Cre recombinase at early stages of development. The DDHD2 ${ }^{-1-}$ mice were backcrossed to C57BL/6J for 10 generations.

\section{Analysis of lower limb motor function in mice}

We evaluated the motor function of lower limbs in mice as described previously ${ }^{43}$. For clasping analysis, mice were suspended by the tail above an open cage for $30 \mathrm{~s}$ for 10 trials. More than 10 animals were analyzed for the characterization of each phonotype. For the extension reflex test, mice were suspended by the tail for $30 \mathrm{~s}$, and then analyzed using the three-point score system. Score 0: bilateral hind limbs were completely crossed, score 1: bilateral hind limbs were of $<90^{\circ}$ at an angle as to the vertical plane, score 2: the unilateral hind limbs were of $<90^{\circ}$ at an angle as to the vertical plane, and score 3: bilateral hind limbs were extended about $120^{\circ}$.

\section{Immunostaining of frozen sections}

Mice were subjected to perfusion fixation with $4 \%$ paraformaldehyde in phosphate-buffered saline (PBS). After the perfusion fixation, spinal cords were isolated from the mice, and then sequentially immersed in $10 \%$ sucrose, $20 \%$ sucrose, and 30\% sucrose solutions. The spinal cords were frozen in O.C.T. compound, and then cut with a microtome into $10-\mu \mathrm{m}$ thick sections. The frozen sections were incubated with $0.2 \%$ TritonX-100 in PBS for membrane permeabilization, blocked with $2 \%$ bovine serum albumin (BSA) in PBS for $1 \mathrm{~h}$ at room temperature, and then incubated with a primary antibody in $2 \% \mathrm{BSA}$ in PBS overnight at $4{ }^{\circ} \mathrm{C}$. The next day, the frozen sections were incubated with a secondary antibody in $2 \% \mathrm{BSA}$ in PBS for $45 \mathrm{~min}$ at room temperature, mounted on a glass slide and analyzed. 


\section{Hematoxylin and eosin staining of spinal cord sections}

Hematoxylin and eosin staining were performed according to the protocols of Abnova. The number of motor neurons in an arbitrarily chosen microscopic field with a size of $124 \times 124 \mu \mathrm{m}^{2}$ was determined. In each mouse, six fields were analyzed, and three mice were used for each experiment.

\section{Culture of primary motor neurons from spinal cords and brains of embryonic mice}

Mice embryos were isolated from pregnant mice at E14.5 or P1. Spinal cords and brains were dissected out and transferred to PBS. The spinal cords and neurons were incubated with a papain/EDTA solution for $10 \mathrm{~min}$ at $37^{\circ} \mathrm{C}$ and transferred through a $40-\mu \mathrm{m}$ cell strainer to $50-\mathrm{ml}$ tubes including primary neuron medium (D-MEM/ F12 containing 20\% FCS, $50 \mathrm{IU} / \mathrm{ml}$ penicillin, $50 \mu \mathrm{g} / \mathrm{ml}$ streptomycin, and N2 and B27 supplements). After centrifugation, the precipitated cells were suspended in the medium and plated on six-well plates with glass coverslips precoated with poly-L-lysine $\left(2 \times 10^{5}\right.$ cells/well). After one day, the culture medium was replaced with medium containing 2\% FCS. After culture for 2 or 7 days, the cells were fixed and immunostained with an antibody against SMI32 or MAP2.

\section{Assessment of ROS production}

To detect ROS production, cells were incubated with $2.5 \mu \mathrm{M}$ CellROX Green for $30 \mathrm{~min}$ at $37^{\circ} \mathrm{C}$. To detect mitochondrial ROS production, cells were incubated with $5 \mu \mathrm{M}$ MitoSOX for $10 \mathrm{~min}$.

\section{Detection of lipid peroxidation}

Cells were incubated with $2 \mu \mathrm{M}$ BODIPY 581/591 C11 for $30 \mathrm{~min}$ at $37^{\circ} \mathrm{C}$. The cells were washed with medium prior to live-cell imaging. Fluorescence of peroxidized species (excitation at $473 \mathrm{~nm}$ ) and non-oxidized species (excitation at $559 \mathrm{~nm}$ ) was recorded. Alternatively, cells were incubated with $0.1 \mu \mathrm{M}$ MitoPeDPP for $15 \mathrm{~min}$ at $37^{\circ} \mathrm{C}$, and MitoPeDPP fluorescence was detected.

To measure the MDA content, brains from 6-monthold mice were homogenized in HB buffer (10 mM Hepes, $220 \mathrm{mM}$ mannitol, and $0.07 \mathrm{M}$ sucrose, $\mathrm{pH} 7.4)$. The homogenate was centrifuged at $800 \times g$ at $4{ }^{\circ} \mathrm{C}$ for $10 \mathrm{~min}$, and then the supernatant was centrifuged at $12,000 \times g$ at $4{ }^{\circ} \mathrm{C}$ for $10 \mathrm{~min}$ to pellet mitochondria. The mitochondrial MDA amount was measured using a TBARS assay kit (Cayman: No.10009055) according to the manufacturer's protocol.

\section{LD detection in MEFs}

Cells were incubated with $1 \mu \mathrm{M}$ LipiDye (Funakoshi) for $30 \mathrm{~min}$, and then fixed. LipiDye fluorescence was detected.

\section{TAG measurement}

TAG was measured using a triglyceride quantification colorimetric kit (Bio Vision: No. K662) according to the manufacturer's protocol.

\section{Measurement of mitochondrial CLs by NAO}

Cells were incubated with $1 \mu \mathrm{M}$ NAO (Thermo Fisher Scientific) for $30 \mathrm{~min}$, and then fixed.

\section{$\mathrm{CL}$ analysis using LC-MS/MS}

Lipids were extracted from cultured cells with the Bligh-Dyer method ${ }^{44}$. An aliquot of the lower (organic) phase was evaporated to dryness under $\mathrm{N}_{2}$, and the residue was dissolved in methanol for LC/MS/MS measurement. LC-electrospray ionization-MS/MS analysis was performed with a Q-Exactive plus mass spectrometry equipped with an UltiMate 3000 LC system (Thermo Fisher Scientific). Lipid samples $(10 \mu \mathrm{l})$ were separated on a Waters CORTECS UPLC C18 column $(1.6 \mu \mathrm{m}$, $2.1 \mathrm{~mm} \times 150 \mathrm{~mm}$ i.d.) at $40{ }^{\circ} \mathrm{C}$ (column oven) using solvent A (acetonitrile/methanol/water (1/1/2, v/v/v) supplemented with $5 \mathrm{mM}$ ammonium formate and $0.05 \%$ ammonium hydroxide) and solvent $\mathrm{B}$ (isopropanol/acetonitrile $(4 / 1, \mathrm{v} / \mathrm{v})$ supplemented with $5 \mathrm{mM}$ ammonium formate and $0.05 \%$ ammonium hydroxide). The following gradient was used: $0 \% \mathrm{~B}(0-2 \mathrm{~min})$, a linear gradient from $0 \% \mathrm{~B}$ to $100 \% \mathrm{~B}$ (2-37 $\mathrm{min}), 100 \% \mathrm{~B}$ (37-49 $\mathrm{min})$, a linear gradient $100 \% \mathrm{~B}$ to $0 \% \mathrm{~B}(49-49.5 \mathrm{~min})$, and $0 \%$ B (49.5-60 min). Flow rate was $220 \mu \mathrm{l} / \mathrm{min}$. CL species were measured by a full scan within $\mathrm{m} / z$ 300-1800 in the negative ion mode, using an Orbitrap Fourier transform mass spectrometry (FTMS) analyzer with a resolution of 70,000. CL (14:0/14:0/14:0/14:0), which is assumed not to occur naturally, was added to samples as an external standard for correction and quantification of CL amount. The mass accuracy was always within $5 \mathrm{ppm}$. The ion spray voltage was set to $-3.2 \mathrm{kV}$. The heated capillary temperature was set at $300{ }^{\circ} \mathrm{C}$. The other parameters were set according to the manufacturer's recommendations. The MS system was controlled with the Xcalibur software.

\section{Measurement of the mitochondrial membrane potential}

Cells were incubated with $2 \mu \mathrm{M}$ JC-1 $\left(5,5^{\prime}, 6,6^{\prime}\right.$-tetrachloro-1,1',3,3'-tetraethylbenzimidazolylcarbocyanine iodide: Thermo Fisher Scientific) for $30 \mathrm{~min}$ at $37^{\circ} \mathrm{C}$, and then washed with medium. Green/Red fluorescence of JC-1 was recorded.

\section{Measurement of ATP}

ATP was measured using a Celltiter-Glo luminescent cell viability assay kit (Promega) according to the manufacturer's protocol. The ATP amount was normalized as to protein concentration. 


\section{Measurement of OCR of MEFs}

Mitochondrial respiratory capacity was measured using an Oxygen Meter Model 781 equipped with a Mitocell MT200 (a closed respiratory chamber) (Strathkelvin Instruments). WT and DDHD2 KO MEFs were cultured in DMEM supplemented with $10 \%$ FCS. The cells were then trypsinized and resuspended at a density of 4.0-8.0 $\times$ $10^{6}$ cells $/ \mathrm{ml}$. Basal respiration was measured in DMEM medium in the presence of $1 \mathrm{mM}$ pyruvate and $24 \mathrm{mM}$ glucose at $37^{\circ} \mathrm{C}$ without $\mathrm{CO}_{2}$ supply. MEFs were subsequently incubated with $1 \mu \mathrm{g} / \mu \mathrm{L}$ oligomycin and $4 \mu \mathrm{M}$ carbonyl cyanide 4-(trifluoromethoxy)phenylhydrazone (FCCP) to measure OCR under conditions blocking ATP synthesis and maximal respiration, respectively.

\section{IF microscopy}

For IF microscopy, cells were fixed with $4 \%$ paraformaldehyde in PBS for 20 min at room temperature, and then permeabilized with $0.2 \%$ Triton X-100 in PBS and blocked with $2 \%$ BSA in PBS. After blocking, the cells were incubated with primary, and then secondary antibodies in $2 \%$ BSA in PBS.

\section{Time-lapse imaging}

Live-cell imaging was performed using an Olympus FluoView 1000 laser scanning microscope. DDHD2 KO MEFs stably expressing DDHD2-WT-mCherry or DDHD2-S351A-mCherry were plated on a glass-bottom dish. The next day, the cells were treated with $0.1 \mathrm{mM} \mathrm{t}$ BHP for $30 \mathrm{~min}$, and then incubated with $0.1 \mu \mathrm{M}$ MitoPeDPP for $15 \mathrm{~min}$. The dish was mounted on the stage of the microscope with a stage-top incubator $\left(37^{\circ} \mathrm{C}, 5 \%\right.$ $\mathrm{CO}_{2}$ ), and live-cell imaging was performed. Time-lapse images were captured at $1 \mathrm{~s}$ intervals for $10 \mathrm{~min}$.

\section{Statistical analysis}

All experiments were performed at least three times. For quantification of fluorescence intensity, at least 30 cells were examined. ImageJ was used for quantification. All results are expressed as means \pm SEM. Statically significant differences were determined using Student's $t$-test. Differences were considered to be significant if the $P$-value was $<0.05$.

\section{Acknowledgements}

We thank Ms. Aya Nagai for her technical assistance and Dr. Hiroshi Doi for the generous gifts of plasmids. We are grateful to Dr. Hiroko Baba, Dr. Tomoko Ishibashi, and the Tagaya lab members for helpful supports and discussions. This work was supported in part by the MEXT-Supported Program for the Strategic Research Foundation at Private Universities (to K.T., Y.M., S.Y., and M.T.) and by a Grant-in-Aid for Scientific Research (JP15H05783 to K.S.) from the Ministry of Education, Culture, Sports, Science and Technology of Japan.

\section{Author details}

'School of Life Sciences, Tokyo University of Pharmacy and Life Sciences, Hachioji, Tokyo 192-0392, Japan. ${ }^{2}$ Division of Regenerative Medicine, Jikei University School of Medicine, 3-25-8 Nishi-Shimbashi, Minato-ku, Tokyo 105-
8461, Japan. ${ }^{3}$ Laboratory of Animal Resources, Center for Disease Biology and Integrative Medicine, Graduate School of Medicine, The University of Tokyo, Tokyo 113-0033, Japan. ${ }^{4}$ Graduate School of Pharmaceutical Sciences, The University of Tokyo, Tokyo 113-0033, Japan. ${ }^{5} J a p a n$ Lipid Technologies LLC, Akita, Akita 010-0825, Japan. ${ }^{6}$ Research Center for Biosignaling, Akita University, Akita 010-8543, Japan. ${ }^{7}$ Present address: Section on Molecular Signal Transduction, Program for Developmental Neuroscience, Eunice Kennedy Shriver NICHD, National Institutes of Health, Bethesda, MD 20892, USA. ${ }^{8}$ Present address: Department of Immunology and Medicine, St. Marianna University School of Medicine, 2-16-1 Sugao, Miyamae, Kawasaki, Kanagawa 216-8511, Japan. ${ }^{9}$ Present address: Teikyo University Institute of Medical Mycology, 359 Otsuka, Hachioji, Tokyo 192-0395, Japan

\section{Author contributions}

T.M. and K.T. conceived the project. T.M., T.B., Y.M., C.H.-M., M.H.-O., Y.E., K.M., N.A., T.T., and S.Y. performed the experiments. T.O.-N. and H.N. performed mass spectrometric analysis. H.J.O. supported the analysis of KO mice. K.N., H.H., and K.S. supported the generation of KO mice. K.T. and M.T. supervised the project. M.T. and T.M. wrote the manuscript.

\section{Conflict of interest}

The authors declare that they have no conflict of interest.

\section{Publisher's note}

Springer Nature remains neutral with regard to jurisdictional claims in published maps and institutional affiliations.

Supplementary Information accompanies this paper at (https://doi.org/ 10.1038/s41419-018-0815-3).

Received: 25 December 2017 Revised: 8 May 2018 Accepted: 21 June 2018 Published online: 23 July 2018

\section{References}

1. Blackstone, C. Cellular pathways of hereditary spastic paraplegia. Annu. Rev. Neurosci. 35, 25-47 (2012).

2. de Souza, P. V. S. et al. Hereditary spastic paraplegia: clinical and genetic hallmarks. Cerebellum 16, 525-551 (2017).

3. Deluca, G. C., Ebers, G. C. \& Esiri, M. M. The extent of axonal loss in the long tracts in hereditary spastic paraplegia. Neuropathol. Appl. Neurobiol. 30, 576-584 (2004).

4. Tani, K., Kogure, T. \& Inoue, H. The intracellular phospholipase $A_{1}$ protein family. Biomol. Concepts 3, 471-478 (2012).

5. Higgs, H. N. \& Glomset, J. A. Purification and properties of a phosphatidic acidpreferring phospholipase $A_{1}$ from bovine testis. J. Biol. Chem. 271, 10874-10883 (1996)

6. Tani, K., Mizoguchi, T., Iwamatsu, A., Hatsuzawa, K. \& Tagaya, M. p125 is a novel mammalian Sec23p-interacting protein with structural similarity to phospholipid-modifying proteins. J. Biol. Chem. 274, 20505-20512 (1999).

7. Nakajima, K. et al. A novel phospholipase $A_{1}$ with sequence homology to a mammalian Sec23p-interacting protein, p125. J. Biol. Chem. 277, 11329-11335 (2002).

8. Higgs, H. N. \& Glomset, J. A. Identification of a phosphatidic acid-preferring phospholipase Al from bovine brain and testis. Proc. Natl Acad. Sci. USA 91, 9574-9578 (1994).

9. Baba, $T$. et al. Phosphatidic acid (PA)-preferring phospholipase $A_{1}$ regulates mitochondrial dynamics. J. Biol. Chem. 289, 11497-11511 (2014).

10. Tesson, C. et al. Alteration of fatty-acid-metabolizing enzymes affects mitochondrial form and function in hereditary spastic paraplegia. Am. J. Hum. Genet. 91, 1051-1064 (2012).

11. Shimoi, W. et al. p125 is localized in endoplasmic reticulum exit sites and involved in their organization. J. Biol. Chem. 280, 10141-10148 (2005).

12. Arimitsu, N. et al. p125/Sec23-interacting protein (Sec23ip) is required for spermiogenesis. FEBS Lett. 585, 2171-2176 (2011).

13. Schuurs-Hoeijmakers, J. H. et al. Mutations in DDHD2, encoding an intracellular phospholipase $A_{1}$, cause a recessive form of complex hereditary spastic paraplegia. Am. J. Hum. Genet. 91, 1073-1081 (2012). 
14. Gonzalez, M. et al. Mutations in phospholipase DDHD2 cause autosomal recessive hereditary spastic paraplegia (SPG54). Eur. J. Hum. Genet. 21, 1214-1218 (2013)

15. Citterio, A. et al. Mutations in CYP2U1, DDHD2 and GBA2 genes are rare causes of complicated forms of hereditary spastic paraparesis. J. Neurol. 261, 373-381 (2014).

16. Magariello, A. et al. Further evidence that DDHD2 gene mutations cause autosomal recessive hereditary spastic paraplegia with thin corpus callosum. Eur. J. Neurol. 21, e25-e26 (2014).

17. Doi, $\mathrm{H}$. et al. Late-onset spastic ataxia phenotype in a patient with a homozygous DDHD2 mutation. Sci. Rep. 4, 7132 (2014).

18. Morikawa, R. K. et al. Intracellular phospholipase $A_{1} \gamma\left(i P\left\llcorner A_{1} \gamma\right)\right.$ is a novel factor involved in coat protein complex 1 - and Rab6-independent retrograde transport between the endoplasmic reticulum and the Golgi complex. J. Biol. Chem. 284, 26620-26630 (2009).

19. Sato, S., Inoue, H., Kogure, T., Tagaya, M. \& Tani, K. Golgi-localized KIAA0725p regulates membrane trafficking from the Golgi apparatus to the plasma membrane in mammalian cells. FEBS Lett. 584, 4389-4395 (2010).

20. Inoue, $\mathrm{H}$. et al. Roles of SAM and DDHD domains in mammalian intracellular phospholipase $A_{1}$ KIAA0725p. Biochim. Biophys. Acta 1823, 930-939 (2012).

21. Baba, T., Yamamoto, A., Tagaya, M. \& Tani, K. A lysophospholipid acyltransferase antagonist, Cl-976, creates novel membrane tubules marked by intracellular phospholipase A 1 KIAA0725p. Mol. Cell. Biochem. 376, 151-161 (2013).

22. Inloes, J. M. et al. The hereditary spastic paraplegia-related enzyme DDHD2 is a principal brain triglyceride lipase. Proc. Natl Acad. Sci. USA 111, 14924-14929 (2014).

23. Aso, C. et al. Protein purification and cloning of diacylglycerol lipase from rat brain. J. Biochem. 159, 585-597 (2016).

24. Araki, M. et al. Enzymatic characterization of recombinant rat DDHD2: a soluble diacylglycerol lipase. J. Biochem. 160, 269-279 (2016).

25. Gotow, T. \& Tanaka, J. Phosphorylation of neurofilament H subunit as related to arrangement of neurofilaments. J. Neurosci. Res. 37, 691-713 (1994).

26. Ludwin, S. K., Kosek, J. C. \& Eng, L. F. The topographical distribution of S-100 and GFA proteins in the adult rat brain: an immunohistochemical study using horseradish peroxidase-labelled antibodies. J. Comp. Neurol. 165, 197-207 (1976).

27. Sarosiek, K. A. et al. BID preferentially activates BAK while BIM preferentially activates BAX, affecting chemotherapy response. Mol. Cell 51, 751-765 (2013).

28. Rosen, D. R. et al. Mutations in Cu/Zn superoxide dismutase gene are associated with familial amyotrophic lateral sclerosis. Nature 362, 59-62 (1993).

29. Hauck, A. K. \& Bernlohr, D. A. Oxidative stress and lipotoxicity. J. Lipid Res. 57 1976-1986 (2016).
30. Nakamura, S. et al. Ionomycin-induced calcium influx induces neurite degeneration in mouse neuroblastoma cells: analysis of a time-lapse live cell imaging system. Free Radic. Res. 50, 1214-1225 (2016).

31. Drummen, G. P., van Liebergen, L. C., Op den Kamp, J. A. \& Post, J. A. C11BODIPY (581/591), an oxidation-sensitive fluorescent lipid peroxidation probe: (micro)spectroscopic characterization and validation of methodology. Free Radic. Biol. Med. 33, 473-490 (2002).

32. Petit, J. M., Maftah, A., Ratinaud, M. H. \& Julien, R. 10N-nonyl acridine orange interacts with cardiolipin and allows the quantification of this phospholipid in isolated mitochondria. Eur. J. Biochem. 209, 267-273 (1992).

33. Welte, M. A. \& Gould, A. P. Lipid droplet functions beyond energy storage. Biochim. Biophys. Acta 1862, 1260-1272 (2017).

34. Herms, A. et al. Cell-to-cell heterogeneity in lipid droplets suggests a mechanism to reduce lipotoxicity. Curr. Biol. 23, 1489-1496 (2013).

35. Bailey, A. P. et al. Antioxidant role for lipid droplets in a stem cell niche of Drosophila. Cell 163, 340-353 (2015).

36. Liu, L. et al. Glial lipid droplets and ROS induced by mitochondrial defects promote neurodegeneration. Cell 160, 177-190 (2015).

37. Liu, L., MacKenzie, K. R., Putluri, N., Maletić-Savatić, M. \& Bellen, H. J. The glianeuron lactate shuttle and elevated ROS promote lipid synthesis in neurons and lipid droplet accumulation in glia via APOE/D. Cell Metab. 26, 719-737 (2017).

38. Yadav, P. K. \& Rajasekharan, R. Misregulation of a DDHD domain-containing lipase causes mitochondrial dysfunction in yeast. J. Biol. Chem. 29, 18562-18581 (2016).

39. Basu Ball, W., Neff, J. K. \& Gohil, V. M. The role of nonbilayer phospholipids in mitochondrial structure and function. FEBS Lett. 592, 1273-1290 (2018).

40. MacDonald, M. L., Murray, I. V. \& Axelsen, P. H. Mass spectrometric analysis demonstrates that BODIPY 581/591 C11 overestimates and inhibits oxidative lipid damage. Free Radic. Biol. Med. 42, 1392-1397 (2007).

41. Liu, P., Jenkins, N. A. \& Copeland, N. G. A highly efficient recombineering-based method for generating conditional knockout mutations. Genome Res. 13, 476-484 (2003).

42. Matsumura, H., Hasuwa, H., Inoue, N., Ikawa, M. \& Okabe, M. Lineagespecific cell disruption in living mice by Cre-mediated expression of diphtheria toxin A chain. Biochem. Biophys. Res. Commun. 32, 275-279 (2004).

43. Magnoni, R. et al. Late onset motoneuron disorder caused by mitochondrial Hsp60 chaperone deficiency in mice. Neurobiol. Dis. 54, 12-23 (2013).

44. Bligh, E. G. \& Dyer, W. J. A rapid method of total lipid extraction and purification. Can. J. Biochem. Physiol. 37, 911-917 (1959).

45. Kamada, S., Kikkawa, U., Tsujimoto, Y. \& Hunter, T. Nuclear translocation of caspase- 3 is dependent on its proteolytic activation and recognition of a substrate-like protein(s). J. Biol. Chem. 280, 857-860 (2005). 